\title{
Disorders of sodium balance and its clinical implications in COVID-19 patients: a multicenter retrospective study
}

\author{
Weihua $\mathrm{Hu}^{1} \cdot$ Xinke $\mathrm{Iv}^{2} \cdot$ Chang $\mathrm{Li}^{3} \cdot$ Yang $\mathrm{Xu}^{4} \cdot$ Yiding $\mathrm{Qi}^{5} \cdot$ Zhuheng Zhang ${ }^{6} \cdot$ Mingxuan $\mathrm{Li}^{5} \cdot$ Feina Cai ${ }^{5} \cdot$ Dan $\mathrm{Liu}^{1}$.

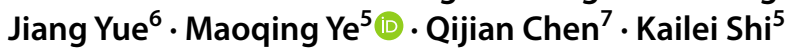

Received: 4 June 2020 / Accepted: 24 September 2020 / Published online: 16 October 2020

(c) Società Italiana di Medicina Interna (SIMI) 2020

\begin{abstract}
Background The worldwide spread of SARS-CoV-2 has infected millions of people leading to over 0.3 million mortalities. The disruption of sodium homeostasis, tends to be a common occurrence in patients with COVID-19.

Methods and results A total of 1,254 COVID-19 patients comprising 124 (9.9\%) hyponatremic patients (under $135 \mathrm{mmol} / \mathrm{L}$ ) and $30(2.4 \%)$ hypernatremic patients (over $145 \mathrm{mmol} / \mathrm{L}$ ) from three hospitals in Hubei, China, were enrolled in the study. The relationships between sodium balance disorders in COVID-19 patients, its clinical features, implications, and the underlying causes were presented. Hyponatremia patients were observed to be elderly, had more comorbidities, with severe pneumonic chest radiographic findings. They were also more likely to have a fever, nausea, higher leukocyte and neutrophils count, and a high sensitivity C-reactive protein (HS-CRP). Compared to normonatremia patients, renal insufficiency was common in both hyponatremia and hypernatremia patients. In addition, hyponatremia patients required extensive treatment with oxygen, antibiotics, and corticosteroids. The only significant differences between the hypernatremia and normonatremia patients were laboratory findings and clinical complications, and patients with hypernatremia were more likely to use traditional Chinese medicine for treatment compared to normonatremia patients. This study indicates that severity of the disease, the length of stay in the hospital of surviving patients, and mortality were higher among COVID-19 patients with sodium balance disorders. Conclusion Sodium balance disorder, particularly hyponatremia, is a common condition among hospitalized patients with COVID-19 in Hubei, China, and it is associated with a higher risk of severe illness and increased in-hospital mortality.
\end{abstract}

Keywords Sodium balance disorders $\cdot$ Hyponatremia $\cdot$ Hypernatremia $\cdot$ SARS-CoV-2 $\cdot$ COVID-19 $\cdot$ Clinical implication

Weihua Hu, Xinke lv, Chang Li and Yang Xu contributed equally to this work.

Maoqing Ye

yemaoqing@fudan.edu.cn

Qijian Chen

ggjycqj@sina.com

Kailei Shi

1879542@qq.com

1 Department of Respiratory, First Hospital of Yangtze University, Clinical Medical College, Yangtze University, Jingzhou 434000, Hubei, China

2 Zhongnan Hospital of Wuhan University, Wuhan 430071, Hubei, China

3 Hubei No.3 People's Hospital of Jianghan University, Wuhan 430033, Hubei, China
4 Department of Orthopedics, Wuhan Children's Hospital (Wuhan Maternal and Child Healthcare Hospital), Tongji Medical College, Huazhong University of Science \& Technology, Wuhan 430015, Hubei, China

5 Department of Cardiology, Huadong Hospital Affiliated To Fudan University, Shanghai 200040, China

6 Wuhan University School of Basic Medical Sciences, Wuhan 430071, Hubei, China

7 Department of Emergency, The fifth Hospital in Wuhan, Wuhan 430050, Hubei, China 


\section{Introduction}

The worldwide spread of Severe Acute Respiratory Syndrome Coronavirus 2 (SARS-CoV-2) [1] infection, the severity and mortality of Coronavirus disease 2019 (COVID-19) have led to a significant threat to international health and the world economy. On 30 January, 2020, the World Health Organization (WHO) declared that the outbreak of SARS-Cov-2 constituted a public health emergency of international concern [2]. SARS-CoV-2 infects human cells by binding to angiotensin I-converting enzyme 2 (ACE2) [3], one of the main anti-regulatory factors of the principal axis of the renin-angiotensin system (RAS) which is an essential factor in controlling blood pressure and electrolyte balance [4]. ACE2 is a cell membrane protein that is widely distributed in different human tissue types, including the epithelial cells of the lung, intestine, kidney, heart, and blood vessels [5, 6]. Binding of SARS-CoV-2 to ACE2 decreases the counter-act of ACE2 on the RAS, which affects electrolyte balance and increases blood pressure [7]. In addition, COVID-19 patients often suffer from gastrointestinal symptoms, such as diarrhea and vomiting [8]. These, however, show that SARS-CoV-2 infection might lead to disruptions in the homeostasis of electrolytes and $\mathrm{pH}$ in vivo.

Sodium disorders are the most common electrolyte disturbances in clinical medicine. Water and sodium balance are regulated independently by specific pathways that are designed to prevent large changes in plasma osmolality (which is primarily determined by the plasma sodium concentration) and the effective circulating volume. The basic principles involved in these two largely separate pathways are: osmoregulation, which maintains the plasma sodium concentration by affecting water excretion through antidiuretic hormone $(\mathrm{ADH})$ and water intake; and volume regulation, which maintains tissue perfusion by affecting sodium excretion through the renin-angiotensin aldosterone system and natriuretic hormones. With the exceptions of decreased effective circulating volume (which will stimulate $\mathrm{ADH}$ at the expense of osmolality), these hormonal pathways do not overlap. The differences between these pathways can be appreciated by considering the clinical manifestations of impaired regulation: hyponatremia (too much water), hypernatremia (too little water), volume expansion (too much sodium), volume depletion (too little sodium) and polyuria. Most importantly, the plasma sodium concentration is regulated by changes in water balance and not by changes in sodium or volume balance. Severe sodium disorders are associated with patients' morbidity and mortality [9], even mild hyponatremias, especially when it complicates conditions, such as heart failure $[10,11]$. Recent experimental studies in rat models have reported the downregulation of ACE2 receptor tissue expression, following a high dietary sodium intake [7, 12]. A clinical study carried out by Aggarwal et al. reported hyponatremia to be much common (50\%) amongst hospitalized COVID-19 patients in the United States [13], and recently study further suggested that serum sodium concentration was inversely correlated with IL-6, and hyponatremia was associated with a more severe outcome of COVID-19 disease [14]. The severity of COVID-19 has been attributed to lower sodium, potassium, and calcium serum concentration levels [15]. All these studies, however, imply that sodium balance disorders have a considerable impact on the therapeutic outcomes of patients with COVID-19 [16]. The associative disorders of serum sodium balance, their clinical characteristics, severity, and outcomes in SARS-CoV-2 infected patients have not been established.

In this study, we determined the varying degrees of sodium imbalance among patients with COVID-19 and identified the association between this imbalance and clinical features, such as in-hospital mortality, length of stay, discharge, and hospital complications. It was revealed that disease severity, the length of hospital stay for surviving patients, and mortality were high among COVID-19 patients with sodium balance disorders.

\section{Methods}

\section{Study participants}

The study included a total of 1,254 COVID-19 patients (First Hospital of Yangtze University, Fifth Hospital in Wuhan, and The Third People's Hospital of Jianghan University) were enrolled from January 27 to March 28, 2020. All the patients were $\geq 18$ years of age and had been diagnosed with COVID-19 according to the Guidance for Coronavirus disease 2019 ( 7 th edition) criteria that were released by the National Health Commission of China [17]. Diagnosed patients were classified to be either mildly, moderately, severely, or critically ill. Patients were treated according to the set treatment protocols released by the National Health Commission of China.

\section{Study design and data collection}

This was a retrospective study conducted during the COVID19 pandemic in Hubei, China. The epidemiological data, laboratory results, demographic, and clinical records of patients during hospitalization were extracted from the electronic medical records using a standardized data collection form. Patients were classified into three distinctive groups according to their serum sodium levels, hyponatremia (under 
$135 \mathrm{mmol} / \mathrm{L})$, normal natremia (135-145 $\mathrm{mmol} / \mathrm{L})$ and hypernatremia (over $145 \mathrm{mmol} / \mathrm{L}$ ).

This research confirmed with the Helsinki Declaration of 1964, as revised in 2013, concerning human rights. Ethical approval was obtained from the Ethical Commission of First Hospital of Yangtze University, Clinical medical college, Yangtze University, Jingzhou, Hubei (K20200305). A written informed consent was waived by the Ethics Commission of the designated hospital for emerging infectious diseases.

\section{Statistical analysis}

Continuous measurements with normal distribution were presented as mean and standard deviation (SD), while those that were not normally distributed were described in median (M) and interquartile range (IQR). Categorical variables, such as onsets of abnormal laboratory values, were described in counts of events and percentages. For continuous variables, single-factor analysis of variance (One-Way ANOVA) was used when normality (and homogeneity of variance) assumptions were satisfied, otherwise, the $K$ Independent Sample Test of Nonparametric Test (Kruskal-Wallis Test) was used. Chi-square test and Fisher's exact test were used in the analysis of categorical variables. A Kaplan-Meier plot was used for survival data. Statistical significance was set at $p \leq 0.05$. The SPSS 23.0 software was used for all analyses.

\section{Results}

\section{Patient characteristics}

A total of 1,254 COVID-19 patients [641 males (51.1\%) and 613 females (48.9\%), median age 56 years old (range, 19-94)] in Hubei province, China were enrolled (Table 1). The majority of these patients $(91.5 \%)$ had either been exposed to an infected family member(s) (7\%), or the pandemic area, Wuhan (84.5\%). The admitted patients' clinical characteristics were fever (71.3\%), cough (56.8\%), fatigue (26.9\%), expectoration (14.4\%), and shortness of breath (20.7\%). Hypertension (27.5\%) was the most common coexisting clinical condition observed. However, a total of 15 patients (1.2\%) had not exhibited any symptoms at the time of hospitalization (Table 1). Of clinical relevance, $5.7 \%$ and $2.1 \%$ of the patients admitted had coronary heart disease (CAD) and heart failure, respectively. Furthermore, a total of 95 patients (7.6\%), 29 patients (2.3\%), 184 patients (14.7\%), 39 patients (3.1\%), 18 patients (1.4\%), and 24 patients (1.9\%) had hyperlipidemia, chronic obstructive pulmonary disease (COPD), type II-diabetes (2-DM), chronic kidney failure (CKD), chronic liver disease, and cancer, respectively. While a total of 153 patients (12.2\%) had other comorbidities.
Compared to normonatremia patients, hyponatremia patients were much older (M[range], 64 [27-94] vs 55 [19-89] years old). In addition, patients in the hyponatremia group were more likely to have a fever ( 80.6 vs $70.0 \%)$ and nausea/vomiting ( 7.3 vs $2.9 \%$ ) (Table 1 ). There were no differing demographic and clinical features between hypernatremia and normonatremia patients. There were no statistical differences in patients' history of exposure to infected family members or the epidemic area (Wuhan, Hubei) among all groups. Comorbidities, such as hypertension (37.9 vs $26.1 \%$ ), CAD (14.5 vs $4.7 \%$ ) and 2-DM (25.0 vs $13.3 \%$ ), were observed more common among patients with hyponatremia compare with normonatremia (all $P<0.05$ ) (Table 1). However, no significant differences in comorbidities differences between hypernatremia and normonatremia patient groups were observed.

\section{Blood test results and radiographic findings}

The patient's median (IQR) blood test levels as listed in Table 2 were within the normal ranges. A total of 180 patients (14.6\%) had 0-2 lobes pneumonia according to computed tomography (CT) findings, while a total of 1071 patients (85.4\%) had multiple mottling, ground-glass opacity or both over the 3 lobes of their lung (Table 1). These findings are shown in Tables 1 and 2, respectively.

Compared to the normonatremia group, patients in the hyponatremia group exhibited a high median leukocyte (M[IQR], 5815 [3340] vs 5400 [2820] cells $/ \mu \mathrm{L})$, and neutrophils count (M[IQR], 4560 [3400] vs 3460 [2490] cells/ $\mu \mathrm{L}$ ), and a lower median lymphocyte counts (M[IQR], 705 [450] vs $1180[810]$ cells $/ \mu \mathrm{L}$ ), eosinophils counts (M[IQR], 10 [30] vs 30 [90] cells $/ \mu \mathrm{L}$ ), and platelet counts (M[IQR], 172 [98.5] vs 196 [102] cells/109/L). There were significant differences in each case (all $p<0.05$, Table 1). Patients in the hyponatremia group exhibited fewer 0-2 lobes lung pneumonia ( 6.5 vs $15.8 \%$ ), but more $>3-5$ lobes lung pneumonia ( 93.5 vs $84.2 \%$ ) when compared to normonatremia patients.

\section{Laboratory findings}

Table 2 shows that the median laboratory IQR findings for HS-CRP, procalcitonin, coagulation index, serum protein, blood lipid, renal indicators, hepatic indicators, cardiac indicators, and electrolyte levels were all within the normal ranges. Compared to the normonatremia group, patients with hyponatremia exhibited higher HS-CRP, procalcitonin, fibrinogen, D-Dimer, fibrinogen degradation products, direct bilirubin, glutamic oxaloacetylase, glutamyltranspeptidase, urea nitrogen, creatinine, creatine kinase (CK), creatine phosphokinase MB (CK-MB), lactate dehydrogenase, and a lower median total protein, albumin, prealbumin, total cholesterol, high-density 
Table 1 Epidemiologic characteristics, clinical features, comorbidities and radiographic findings of patients stratified by three different levels of serum sodium

\begin{tabular}{|c|c|c|c|c|c|c|c|}
\hline \multirow[t]{2}{*}{ Attribute } & \multirow[t]{2}{*}{ Total $(n=1254)$} & \multicolumn{3}{|c|}{ Serum bloood sodium } & \multicolumn{3}{|l|}{$p$ value } \\
\hline & & $\begin{array}{l}\text { Hyponatremia } \\
(n=124) \\
(<135 \mathrm{mmol} / \mathrm{L})\end{array}$ & $\begin{array}{l}\text { Normonatremia } \\
(n=1100)(135- \\
145 \mathrm{mmol} / \mathrm{L})\end{array}$ & $\begin{array}{l}\text { Hyperna- } \\
\text { tremia }(n=30) \\
(>145 \mathrm{mmol} / \mathrm{L})\end{array}$ & p1 (1-2) & p2 (2-3) & p3 $(1-3)$ \\
\hline No. of patients (\%) & $1254(100)$ & $124(9.9)$ & $1100(87.7)$ & $30(2.4)$ & - & - & - \\
\hline $\begin{array}{l}\text { Age, median(range), } \\
\text { year }\end{array}$ & $56(19-94)$ & $64(27-94)$ & $55(19-89)$ & $62(29-84)$ & $<0.001 *$ & 0.186 & 1.000 \\
\hline \multicolumn{8}{|l|}{ Gander } \\
\hline Male & $641(51.1)$ & $76(61.3)$ & $550(50.0)$ & $15(50.0)$ & $0.018^{*}$ & 1.000 & 0.259 \\
\hline Female & $613(48.9)$ & $48(38.7)$ & $550(50.0)$ & $15(50.0)$ & & & \\
\hline \multicolumn{8}{|c|}{ Exposure or contact information, no.(\%) } \\
\hline Family source & $89(7.0)$ & $4(3.2)$ & $82(7.5)$ & $3(10.0)$ & 0.131 & 0.547 & 0.128 \\
\hline Exposure to Wuhan & $1059(84.5)$ & $106(85.5)$ & $927(84.2)$ & $26(86.7)$ & & & \\
\hline Others & $106(8.5)$ & $14(11.3)$ & $91(8.3)$ & $1(3.3)$ & & & \\
\hline \multicolumn{8}{|c|}{ Signs and symptoms at disease onset, no.(\%) } \\
\hline Fever & $894(71.3)$ & $100(80.6)$ & $770(70.0)$ & $24(80.0)$ & $0.013^{*}$ & 0.237 & 0.936 \\
\hline Cough & $712(56.8)$ & $71(57.3)$ & $630(57.3)$ & $16(53.3)$ & 0.998 & 0.667 & 0.697 \\
\hline Expectoration & $180(14.4)$ & $21(16.9)$ & $157(14.3)$ & $2(6.7)$ & 0.425 & 0.237 & 0.157 \\
\hline Fatigue & $337(26.9)$ & $39(31.5)$ & $292(26.5)$ & $6(20.0)$ & 0.243 & 0.422 & 0.216 \\
\hline Shortness of breath & 259 (20.7) & $30(24.2)$ & $220(20.0)$ & $9(30.0)$ & 0.272 & 0.179 & 0.512 \\
\hline Nausea and vomiting & $42(3.3)$ & $9(7.3)$ & $32(2.9)$ & $1(3.3)$ & $0.011^{*}$ & 0.892 & 0.711 \\
\hline Diarrhea & $109(8.7)$ & $12(9.7)$ & $94(8.5)$ & $3(10.0)$ & 0.671 & 0.960 & 0.772 \\
\hline Chills & $70(5.6)$ & $11(8.9)$ & $59(5.4)$ & 0 & & & \\
\hline Pharyngalgia & $47(3.7)$ & $1(0.8)$ & $44(4.0)$ & $2(6.7)$ & 0.124 & 0.794 & 0.178 \\
\hline Headache & $47(3.7)$ & $6(4.8)$ & $41(3.7)$ & 0 & & & \\
\hline Chest pain & $37(3.0)$ & $4(1.7)$ & $31(2.8)$ & $2(6.7)$ & 0.979 & 0.493 & 0.728 \\
\hline Asymptomatic & $15(1.2)$ & 0 & $15(1.4)$ & 0 & & & \\
\hline \multicolumn{8}{|l|}{ Comorbidity, no.(\%) } \\
\hline Hypertension & $345(27.5)$ & 47 (37.9) & $287(26.1)$ & $11(36.7)$ & $0.005^{*}$ & 0.195 & 0.900 \\
\hline $\mathrm{CAD}$ & $72(5.7)$ & $18(14.5)$ & $52(4.7)$ & $2(6.7)$ & $0.000009 *$ & 0.623 & 0.251 \\
\hline Hyperlipidimia & 95 (7.6) & $7(5.6)$ & $85(7.7)$ & $3(10)$ & 0.404 & 0.647 & 0.385 \\
\hline Heart failure & $26(2.1)$ & $5(4.0)$ & $20(1.8)$ & $1(3.3)$ & 0.098 & 0.937 & 0.728 \\
\hline COPD & $29(2.3)$ & $5(4.0)$ & $23(2.1)$ & $1(3.3)$ & 0.17 & 0.860 & 0.727 \\
\hline 2-DM & $184(14.7)$ & $31(25)$ & $146(13.3)$ & $7(23.3)$ & $0.0004 *$ & 0.112 & 0.849 \\
\hline CKD & $39(3.1)$ & $7(5.6)$ & $31(2.8)$ & $1(3.3)$ & 0.085 & 0.697 & 0.957 \\
\hline CLD & $18(1.4)$ & $2(1.7)$ & $14(1.3)$ & $2(6.7)$ & 0.752 & 0.092 & 0.357 \\
\hline Cancer & $24(1.9)$ & $5(4.0)$ & $18(1.6)$ & $1(3.3)$ & 0.063 & 0.995 & 0.741 \\
\hline Others & $153(12.2)$ & $16(12.9)$ & $130(11.8)$ & $7(23.3)$ & 0.724 & 0.057 & 0.150 \\
\hline \multicolumn{8}{|c|}{ CT findings involving lobes no. (\%) } \\
\hline $0-2$ lobe & $183(14.6)$ & $8(6.5)$ & $174(15.8)$ & $1(3.3)$ & $0.005^{*}$ & 0.108 & 0.826 \\
\hline 3 or more lobes & $1071(85.4)$ & $116(93.5)$ & $926(84.2)$ & $29(96.7)$ & & & \\
\hline
\end{tabular}

*Significant statistical difference

$C A D$ coronary heart disease, $C O P D$ chronic obstructive pulmonary disease, 2-DM type II-diabetes, $C K D$ chronic kidney failure, $C L D$ chronic liver disease, $C T$ Computed tomography

lipoprotein (HDL), low-density lipoprotein (LDL), potassium, chlorine and total calcium, with significant differences in each case (all $p<0.05$, Table 2). Compared to patients with normonatremia, patients with hypernatremia exhibited higher median HS-CRP, PD-Dimer, FDP, LDH, chlorine, and a lower median albumin, prealbumin, globulin, alkaline phosphatase, urea nitrogen, potassium and total calcium, with significant differences in each case (all $p<0.05$, Table 2). 
Table 2 Laboratory findings of patients stratified by three levels of serum sodium

\begin{tabular}{|c|c|c|c|c|c|c|c|}
\hline \multirow[t]{2}{*}{ Attribute, median (IQR) } & \multirow[t]{2}{*}{ Total $(n=1254)$} & \multicolumn{3}{|c|}{ Serum bloood sodium } & \multicolumn{3}{|l|}{$p$ value } \\
\hline & & $\begin{array}{l}\text { Hyponatremia } \\
(n=124) \\
(<135 \mathrm{mmol} / \mathrm{L})\end{array}$ & $\begin{array}{l}\text { Normonatremia } \\
(n=1100)(135- \\
145 \mathrm{mmol} / \mathrm{L})\end{array}$ & $\begin{array}{l}\text { Hyperna- } \\
\text { tremia }(n=30) \\
(>145 \mathrm{mmol} / \mathrm{L})\end{array}$ & $\mathrm{p} 1(1-2)$ & $\mathrm{p} 2(2-3)$ & $\mathrm{p} 3(1-3)$ \\
\hline \multicolumn{8}{|l|}{ Blood tests, median (IQR) } \\
\hline Leukocytes count $\times 10^{6} / \mathrm{L}$ & $5410(3000)$ & $5815(3340)$ & $5400(2820)$ & $5410(5370)$ & $0.031 *$ & 0.259 & \\
\hline Neutrophils count $\times 10^{6} / \mathrm{L}$ & $3580(2620)$ & $4560(3400)$ & $3460(2490)$ & $4290(4180)$ & $<0.001 *$ & 1.000 & 0.177 \\
\hline $\begin{array}{l}\text { Lymphocyte count } \\
\times 10^{6} / \mathrm{L}\end{array}$ & $1110(790)$ & $705(450)$ & $1180(810)$ & $970(650)$ & $<0.001^{*}$ & 0.250 & 0.062 \\
\hline Eosinophils count $\times 10^{6} / \mathrm{L}$ & $30(90)$ & $10(30)$ & $30(90)$ & $15(80)$ & $<0.001 *$ & 0.144 & 1.000 \\
\hline Erythrocyte $\times 10^{6} / \mathrm{L}$ & $4290(710)$ & $4190(840)$ & $4310(700)$ & $4155(770)$ & & & \\
\hline Hemoglobin, g/L & $130(22)$ & $127(25)$ & $130(22)$ & $124(23.75)$ & & & \\
\hline Platelet $\times 10^{9} / \mathrm{L}$ & $195(100.5)$ & $172(98.5)$ & $196(102)$ & $220(90)$ & $0.021 *$ & 0.740 & 0.062 \\
\hline \multicolumn{8}{|c|}{ Laboratory tests, median (IQR) } \\
\hline HS-CRP, mg/L, & $12.1(46.3)$ & $62(87.875)$ & $9.1(36.72)$ & $53(89.6)$ & $<0.001 *$ & $0.002 *$ & 0.598 \\
\hline Procalcitonin, ng/ml, & $0.05(0.1)$ & $0.11(0.17)$ & $0.045(0.078)$ & $0.069(0.166)$ & $<0.001 *$ & 0.580 & 0.205 \\
\hline Fibrinogen, $g / L$ & $3.61(2.02)$ & $4.47(1.89)$ & $3.45(2.01)$ & $4.77(2.19)$ & $<0.001 *$ & $0.002^{*}$ & 1.000 \\
\hline D-Dimer, ug/L & $0.490(0.890)$ & $0.955(2.21)$ & $0.450(0.770)$ & $1.560(3.72)$ & $<0.001 *$ & $<0.001 *$ & 0.333 \\
\hline FDP, ug/L & $3.330(3.500)$ & $5.31(5.44)$ & $3.09(3.17)$ & $6.95(9.94)$ & $<0.001 *$ & $<0.001 *$ & 0.601 \\
\hline Total bilirubin, $\mathrm{mmol} / \mathrm{L}$ & $10.90(6.50)$ & $11.32(7.40)$ & $10.98(6.33)$ & $8.10(9.90)$ & 0.648 & 0.107 & $0.039 *$ \\
\hline Direct bilirubin, $\mathrm{mmol} / \mathrm{L}$ & $3.70(2.20)$ & $4.30(2.60)$ & $3.60(2.10)$ & $3.00(4.25)$ & $0.001 *$ & 1.000 & 0.095 \\
\hline ALT, U/L & $23.00(24.05)$ & $27.00(30.45)$ & $22.00(23.40)$ & $25.00(34.25)$ & $0.032 *$ & 0.320 & 1.000 \\
\hline GOT, U/L & $28.90(18.50)$ & $39.00(25.30)$ & $28.00(17.42)$ & $31.00(31.50)$ & $<0.001 *$ & 1.000 & 0.104 \\
\hline Total protein, $\mathrm{g} / \mathrm{L}$ & $68.05(9.20)$ & $66.00(8.20)$ & $68.30(9.23)$ & $67.90(8.70)$ & $0.001 *$ & 1.000 & 0.898 \\
\hline Albumin, $\mathrm{g} / \mathrm{L}$ & $38.2(7.80)$ & $35.50(6.30)$ & $38.50(7.70)$ & $33.40(7.05)$ & $<0.001^{*}$ & $<0.001 *$ & 0.297 \\
\hline Prealbumin, mg/L & $164.00(141.38)$ & $95.00(62.00)$ & $179.50(137.80)$ & $107.50(101.75)$ & $<0.001 *$ & $0.005^{*}$ & 0.484 \\
\hline Globulin, g/L & $30.0(7.30)$ & $30.90(7.35)$ & $29.90(7.38)$ & $33.70(7.55)$ & 1.000 & $0.001 *$ & $0.010^{*}$ \\
\hline $\begin{array}{l}\text { Glutamyltranspeptidase, } \\
\text { U/L }\end{array}$ & $27.00(28.00)$ & $34.00(43.70)$ & $26.00(27.95)$ & $26.00(20.50)$ & $0.012^{*}$ & 1.000 & 0.276 \\
\hline $\begin{array}{l}\text { Alkaline phosphatase, } \\
\text { U/L }\end{array}$ & $59.00(28.00)$ & $60.00(34.00)$ & $59.00(28.00)$ & $52.00(28.00)$ & 0.401 & $0.031 *$ & $0.023^{*}$ \\
\hline Urea nitrogen, umol/L & $4.10(2.30)$ & $4.88(2.95)$ & $4.08(2.10)$ & $5.90(7.00)$ & $0.002^{*}$ & $0.007 *$ & 0.780 \\
\hline Creatinine, umol/L & $64.00(25.00)$ & $69.00(26.60)$ & $63.05(25.00)$ & $68.00(36.50)$ & $0.002 *$ & 0.151 & 1.000 \\
\hline Uric acid, umol/L & $261.00(135.00)$ & $253.00(137.25)$ & $261.00(133.50)$ & $275.00(193.00)$ & 0.361 & 0.772 & 0.893 \\
\hline Total cholesterol, umol/L & $3.99(1.30)$ & $3.61(1.01)$ & $4.04(1.40)$ & $3.70(1.58)$ & $<0.001 *$ & 0.241 & 1.000 \\
\hline Triglyceride, umol/L & $1.21(0.81)$ & $1.17(0.64)$ & $1.22(0.85)$ & $1.425(1.13)$ & 0.528 & 0.052 & $0.033^{*}$ \\
\hline $\mathrm{HDL}, \mathrm{umol} / \mathrm{L}$ & $1.01(0.39)$ & $0.95(0.42)$ & $1.02(0.40)$ & $0.875(0.36)$ & $0.032 *$ & 0.153 & 1.000 \\
\hline LDL, umol/L & $2.48(1.12)$ & $2.15(0.94)$ & $2.50(1.13)$ & $2.50(1.27)$ & $0.001^{*}$ & 1.000 & 0.761 \\
\hline Homocysteine, umol/L & $7.70(3.50)$ & $8.20(5.45)$ & $7.70(3.45)$ & $7.20(5.48)$ & 0.108 & 0.364 & 0.091 \\
\hline $\mathrm{CK}, \mathrm{U} / \mathrm{L}$ & $67.00(68.5)$ & $92.00(110.00)$ & $64.00(65.00)$ & $71.50(126.50)$ & $<0.001 *$ & 0.692 & 0.553 \\
\hline CK-MB, U/L & $9.22(6.55)$ & $11.00(10.42)$ & $9.10(5.63)$ & $8.55(17.10)$ & $0.016^{*}$ & 1.000 & 0.937 \\
\hline Hs-cTnI, ng/ml & $0.01(0.00)$ & $0.01(0.00)$ & $0.01(0.00)$ & $0.01(0.01)$ & 0.664 & & \\
\hline $\mathrm{LDH}, \mathrm{U} / \mathrm{L}$ & $206.50(11.75)$ & $287.55(169.00)$ & 200.00 (99.00) & $278.50(206.25)$ & $<0.001^{*}$ & $0.001 *$ & 1.000 \\
\hline Potassium, mmol/L & $3.90(0.62)$ & $3.755(0.73)$ & $3.90(0.64)$ & $3.65(0.65)$ & $0.004 *$ & $0.019 *$ & 0.946 \\
\hline Chlorine, $\mathrm{mmol} / \mathrm{L}$ & $104.05(5.47)$ & $99.00(5.50)$ & $104.90(5.00)$ & $109.70(5.65)$ & $<0.001 *$ & $<0.001^{*}$ & $<0.001^{*}$ \\
\hline Total calcium, mmol/L & $2.11(0.21)$ & $2.03(0.17)$ & $2.12(0.20)$ & $2.00(0.19)$ & $<0.001 *$ & $0.001^{*}$ & 1.000 \\
\hline
\end{tabular}

*Significant statistical difference

$H S$-CRP High sensitivity C-reactive protein, FDP Fibrinogen degradation products, ALT Alanine aminotransferase, GOT Glutamic oxaloacetate, $H D L$ High-density lipoprotein, $L D L$ Low-density lipoprotein, $C K$ Creatine kinase, $C K-M B$ Creatine phosphokinase MB, $H s-c T n I$ High sensitivity troponin, $L D H$ Lactate dehydrogenase 
Table 3 Complications and therapy of patients stratified by three levels of serum sodium

\begin{tabular}{|c|c|c|c|c|c|c|c|}
\hline \multirow[t]{2}{*}{ Attribute } & \multirow[t]{2}{*}{ Total $(n=1254)$} & \multicolumn{3}{|c|}{ Serum bloood sodium } & \multicolumn{3}{|l|}{$p$ value } \\
\hline & & $\begin{array}{l}\text { Hyponatremia } \\
(n=124) \\
(<135 \mathrm{mmol} / \mathrm{L})\end{array}$ & $\begin{array}{l}\text { Normonatremia } \\
(n=1100)(135- \\
145 \mathrm{mmol} / \mathrm{L})\end{array}$ & $\begin{array}{l}\text { Hyperna- } \\
\text { tremia }(n=30) \\
(>145 \mathrm{mmol} / \mathrm{L})\end{array}$ & p1 (1-2) & p2 (2-3) & p3 (1-3) \\
\hline \multicolumn{8}{|l|}{ Complications } \\
\hline Renal insufficiency & $48(3.8)$ & $9(7.3)$ & $35(3.2)$ & $4(13.3)$ & $0.021 *$ & $0.012 *$ & 0.479 \\
\hline Hepatic dysfunction & $282(22.5)$ & $36(29.0)$ & $238(21.6)$ & $8(26.7)$ & 0.061 & 0.649 & 0.651 \\
\hline Anemia & $33(2.6)$ & $6(4.8)$ & $27(2.5)$ & 0 & 0.120 & - & - \\
\hline $\begin{array}{l}\text { Acute myocardial } \\
\text { infarction }\end{array}$ & $4(0.3)$ & $2(1.6)$ & $2(0.2)$ & 0 & 0.069 & - & - \\
\hline $\begin{array}{l}\text { Hypoproteinemia } \\
\quad(<30 \mathrm{~g} / \mathrm{L})\end{array}$ & $91(7.3)$ & $13(10.5)$ & $73(6.6)$ & $5(16.7)$ & 0.112 & $0.032^{*}$ & 0.344 \\
\hline \multicolumn{8}{|l|}{ Treatment, no. (\%) } \\
\hline Treated with oxygen & $800(63.8)$ & $108(87.1)$ & $670(60.1)$ & $22(73.3)$ & $<0.001^{*}$ & 0.168 & 0.062 \\
\hline $\begin{array}{l}\text { Oxygen inhalation } \\
\text { through nasal duct }\end{array}$ & $553(44.1)$ & $57(46.0)$ & $481(43.7)$ & $15(50.0)$ & $<0.001 *$ & 0.168 & 0.220 \\
\hline $\begin{array}{l}\text { Oxygen absorption by } \\
\text { mask }\end{array}$ & $162(12.9)$ & $31(25.0)$ & $128(11.6)$ & $3(10.0)$ & & & \\
\hline $\begin{array}{l}\text { High flux oxygen } \\
\text { absorption }\end{array}$ & $10(0.8)$ & $2(1.6)$ & $7(0.6)$ & $1(3.3)$ & & & \\
\hline Noninvasive ventilator & $65(5.2)$ & $16(12.9)$ & $46(4.2)$ & $3(10.0)$ & & & \\
\hline Invasive ventilator & $10(0.8)$ & $2(1.6)$ & $8(0.7)$ & 0 & & & \\
\hline $\begin{array}{l}\text { Without oxygen treat- } \\
\text { ment }\end{array}$ & $454(37.2)$ & $16(12.9)$ & $430(39.1)$ & $8(26.7)$ & & & \\
\hline Antiviral drugs & $1158(92.3)$ & $118(95.2)$ & $1012(92.0)$ & $28(93.3)$ & 0.210 & 0.940 & 0.957 \\
\hline Antibiotic drugs & $1076(85.8)$ & $115(92.7)$ & $934(84.9)$ & $27(90.0)$ & $0.018 *$ & 0.609 & 0.902 \\
\hline Corticosteroids & $503(40.1)$ & $72(58.1)$ & $422(38.4)$ & $9(30.0)$ & $0.00002^{*}$ & * 0.352 & $0.006^{*}$ \\
\hline $\begin{array}{l}\text { Traditional Chinese } \\
\text { medicine }\end{array}$ & $1029(82.1)$ & $101(81.5)$ & $908(82.5)$ & $20(66.7)$ & 0.762 & $0.025^{*}$ & 0.077 \\
\hline \multicolumn{8}{|l|}{ Disease severity, no.(\%) } \\
\hline Mild type & $104(8.3)$ & $3(2.4)$ & $100(9.1)$ & $1(3.3)$ & $<0.001 *$ & $0.038^{*}$ & 0.900 \\
\hline Moderate type & $866(69.1)$ & $63(50.1)$ & $786(71.4)$ & $17(56.7)$ & & & \\
\hline Severe type & $200(15.9)$ & $35(28.2)$ & $157(14.3)$ & $8(26.7)$ & & & \\
\hline Critical type & $84(6.7)$ & $23(18.5)$ & $5(5.2)$ & $4(13.3)$ & & & \\
\hline \multicolumn{8}{|c|}{ Length of stay, days, median (IQR) } \\
\hline $\begin{array}{l}\text { Survived patients, } \\
\text { no.\% }\end{array}$ & $1160(92.5)$ & $104(83.9)$ & $1031(93.7)$ & $25(83.3)$ & - & - & - \\
\hline Surviving patients & $17.5(13.0)$ & $20.0(16.0)$ & $17.0(13.0)$ & $17.0(14.5)$ & $0.022^{*}$ & 1.000 & 0.796 \\
\hline Dead patients & $7.0(8.0)$ & $6.0(10.8)$ & $8.0(8.0)$ & $5.0(14.5)$ & 0.618 & 0.884 & 0.668 \\
\hline \multicolumn{8}{|l|}{ Outcome, no.(\%) } \\
\hline Recovery & $1160(92.5)$ & $104(83.9)$ & $1031(93.7)$ & $25(83.3)$ & $0.0006^{*}$ & $0.023 *$ & 0.943 \\
\hline Death & $94(7.5)$ & $20(16.1)$ & $69(6.3)$ & $5(16.7)$ & & & \\
\hline
\end{tabular}

*Significant statistical difference

\section{Complications and therapeutic management of patients}

Out of the total 1254 patients, $22.5 \%$ had hepatic dysfunction, $7.3 \%$ had hypoproteinemia, 3.8\% had renal insufficiency, $2.6 \%$ had anemia, while $0.3 \%$ had an acute myocardial infarction (Table 3). A total of 800 patients (63.8\%) were treated with oxygen, the total percent of the use of inhaled oxygen, oxygen absorption by mask, high-influx oxygen absorption, and non-invasive and invasive ventilation was $44.1,12.9,0.8,5.2$ and $0.8 \%$, respectively. The proportion of antiviral therapy used was the highest (92.3\%), followed by antibiotic therapy (85.8\%), Traditional Chinese Medicine (82.1\%) and corticosteroids (40.1\%) (Table 3).

Renal insufficiency was the most common complication among patients with hyponatremia when compared 
to normonatremia patients ( 7.3 vs $3.2 \%$ ). However, renal insufficiency (13.3 vs $3.2 \%$ ) and hypoproteinemia (16.7 vs $6.6 \%$ ) were the most common complication observed among patients with hypernatremia. In addition, hyponatremia patients required intensive oxygen treatment compared to normonatremia patients (87.1 vs $60.1 \%$ ) including the use of oxygen inhalation, oxygen absorption by mask, high-influx oxygen absorption, and non-invasive and invasive ventilation (Table 3). The therapeutic use of antibiotics (92.7 vs $84.9 \%$ ) and corticosteroids (58.1 vs $38.4 \%$ ) was significantly high in hyponatremia patients compared to normonatremia patients $(p<0.05$; Table 3$)$. Of clinical importance, the use of Traditional Chinese Medicine for treatment in hypernatremia patients was significantly high compared to normonatremia patients $(66.7$ vs $82.5 \%)(\mathrm{p}<0.05$; Table 3$)$.

\section{Association between sodium balance disorders with disease severity and outcome in patients with COVID-19}

Diagnosed patients were classified to be either mildly, moderately, severely, or critically ill. From the 1254 patients, 104 (8.3\%) patients were mild cases, 866 (69.1\%) patients were moderate cases, and $200(15.9 \%)$ patients were severe cases, while $84(6.7 \%)$ patients were critically ill. During follow-up, a total of $94(7.5 \%)$ patients died, while a total of $1160(92.5 \%)$ patients recovered. The median hospital stay for patients who survived was 17.5 days (IQR 13.0) (Table 3). Compared to the normonatremia group, disease severity was high in patients with sodium balance disorders. A total of 66 (53.2\%) hyponatremia patients, and 18 (60.0\%) hypernatremia patients exhibited either a mild or a moderate type case, with ratios lower than patients with normonatremia (886 patients, $80.5 \%$ ). However, a total of 58 (46.8\%) hyponatremia patients and 12 (40.0\%) hypernatremia patients exhibited severe or critical illnesses cases with ratios high than patients with normonatremia (214 patients, $19.5 \%$ ). The mortality rate was higher among patients with sodium balance disorders compared to normonatremia $(16.1 / 16.7$ vs $6.3 \% ; P<0.001)$ as shown in Table 3, and in the Kaplan-Meier survival curves in Fig. 1. The length of hospital stay for patients with sodium balance disorders was longer compared to normonatremia patients (M [IQR] 20 [16.0] / 17 [14.5] days vs 17.0 [13.0] days; $\mathrm{p}$ $<0.05)$.

\section{Discussion}

The study included a total of 1,254 patients with SARSCoV-2 infection admitted in three hospitals in Hubei, China, were enrolled. They exhibited abnormal serum sodium levels, including hyponatremia (9.9\%) and hypernatremia (2.4\%). The length of hospital stay for patients who survived, severity and the mortality rate was higher among patients with sodium balance disorders.

The study showed that hyponatremia was common among these patients as compared to hypernatremia (9.9 vs $2.4 \%$ ). In addition, it was revealed that demographic features, clinical features, associated comorbidities, radiographic findings, blood tests, laboratory findings, complications precisely, renal insufficiency play a considerable role between hyponatremia and normonatremia COVID-19 patients. However, of importance, the only apparent significant differences between the hypernatremia and normonatremia patients were laboratory findings and clinical complications. Besides, hyponatremia patients required intensive oxygen support, and were administered with significantly high doses of antibiotics and corticosteroids compared to normonatremia patients. Hypernatremia patients were mostly treated with Traditional Chinese Medicine. Hyponatremia patients had a protracted hospital stay compared to both hypernatremia and
Fig. 1 Kaplan-Meier survival curves of patients with 3 levels of serum sodium

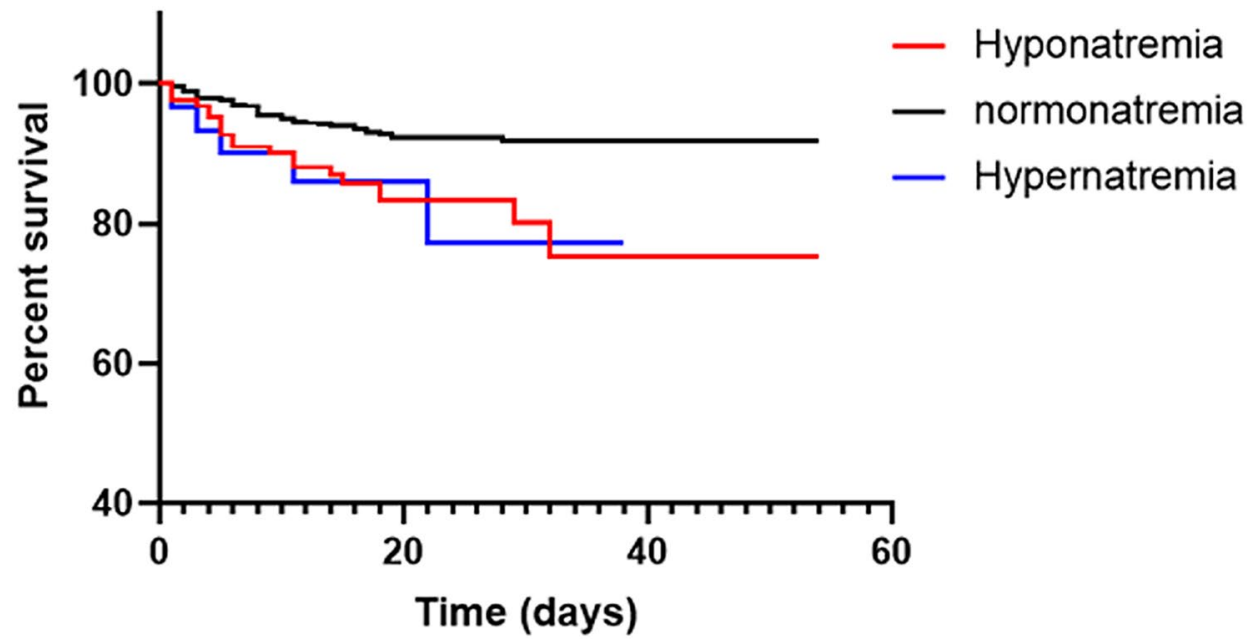


normonatremia patients. However, the hospital stay durations for hypernatremia, and normonatremia patients were not significantly different. This indicates that COVID-19 patients with hyponatremia tended to have a more severe illness, and had poor clinical outcomes compared to hypernatremia patients.

Hyponatremia is divided into euvolemic, hypovolemic, and hypervolemic hyponatremia. Due to the lack of data on urine sodium concentration, we could not stratify our patients into these classes. However, we analyzed the relevant clinical manifestations to evaluate the possible etiopathogenetic classifications of our COVID-19 patients with hyponatremia. Hypovolemic hyponatremia patients have both a total body sodium ion and a water deficit, with the sodium ion deficit exceeding the water deficit. This is common among patients with high gastrointestinal and renal water and solute losses accompanied by free water or hypotonic fluid intake. Previous studies have shown that increased electrolyte gastrointestinal loss renal and insufficiency are the most notable causes of serum sodium disorder $[9,18]$. Only a small proportion of patients in this study exhibited symptoms of gastrointestinal electrolyte loss, these including diarrhea (8.7\%), nausea and vomiting (3.3\%). However, except for cases of hyponatremia, which showed a significant statistical difference in nausea and vomiting (7.2 vs $2.9 \%$ ), there were no significant incidental associative differences between gastrointestinal symptoms and serum sodium disorder. These results showed that gastrointestinal electrolyte loss does not considerably contribute to sodium serum disorders in COVID-19 patients. Likewise, on the other hand, renal insufficiency was highly correlated to hyponatremia and hypernatremia patients, with blood urea nitrogen and creatinine levels were significantly elevated in hyponatremia patients compared to normonatremia patients. In addition, COVID-19 patients with hyponatremia were older and more pluricomorbid. This could be associated with the insufficiency of reserve renal functions in old patients and the decreased abilities of the kidneys to regulate electrolytes in SARS-CoV-2 infections. It has not been established whether renal insufficiency is an etiological factor of serum sodium imbalance in COVID-19 patients.

In hypervolemia hyponatremia, the total body water content is increased more than total body sodium ion occurs, as in congestive heart failure (CHF), nephrotic syndrome, and cirrhosis. In present study, liver function and albumin levels in hyponatremia patients were within normal ranges. This suggested a less likelihood of nephrotic syndrome or cirrhosis. Previous studies had shown mild hyponatremia is associated with poor clinical outcomes as it complicates conditions, such as heart failure $[9,19]$. Because the human cardiac myocytes express ACE2 that acts as a viral receptor, SARS-CoV-2 can also result in heart dysfunction [3]. Hyponatremia in this study showed significant differences on CAD and hypertension ratios when compared to normonatremia patients. This indicated the possibility of hypervolemia hyponatremia due to cardiac dysfunctions. Further investigations of serum sodium disorder in COVID-19 patients are pivotal, considering the clinical implications of a disordered serum sodium level.

Euvolemic hyponatremia is the most common dysnatremia in hospitalized patients. Syndrome of inappropriate antidiuresis (SIADH) is the common symptom of hyponatremia. Viral pneumonia is one of the common causes for SIADH. Recently, a case series of COVID-19-associated SIADH has been documented [20-22]. They showed that treating patients presenting with hyponatremia and fever was a high predictive factor for COVID-19. In addition, the hypothesized mechanisms for SIADH in COVID-19 infection include inflammatory cytokine release, ventilation-perfusion mismatch, intravascular volume depletion, stress, pain, emotions, etc. [20, 23]. Positive-pressure ventilation (PPV) is a well-known reason of SIADH and hyponatremia development [24]. This is probably due to nonosmotic stimulation of antidiuretic hormone (ADH) secretion, as pulmonary venous baroreceptors respond to a reduction in effective blood volume. Therefore, SIADH may be one of the reasons for unsatisfactory treatment of COVID-19 with PPV [23]. Our study adds further evidence for the association of COVID-19 and SIADH in larger cohort study, and we also noticed a high percent of non-invasive ventilation use in hyponatremia patients, suggesting the possible relationship between SIADH and PPV. However, our hyponatremia patients also have higher rate of antibiotics and corticosteroid use, effect of glucocorticoids [25] or antibiotics [26] on SIADH have also been elucidated. These treatments may be causative, but need further investigation.

Some limitations existed in this study. Due to logistical limitations at the beginning of these emerging infectious diseases in Wuhan, some data, such as urine volume, urine sodium and chloride concentration, were not obtained in isolation wards, which limited the classification of hyponatremia patients, and the long-term outcome of the COVID-19 patients relative to serum sodium disorders also unclear. However, the large sample size reflected the fundamental pathophysiological process of COVID-19 in present study, and the results derived from this study are important in the clinical management of COVID-19.

In conclusion, serum sodium imbalance is a common characteristic feature and associated with severe illness, poor clinical outcome(s) and increased in-hospital mortality in COVID-19 patients.

Acknowledgements We acknowledge all health-care workers involved in the diagnosis and treatment of patients in Hubei, China. 
Authors' contributions QC, MY and KS designed the study, maintained data integrity, accuracy and did the data analysis. XL, CL, YX, YQ, $\mathrm{ZZ}$, ML collected and recorded the data. FC, DL and JY did the data analysis. WH, QC, MY and KS drafted the manuscript. All the authors revised the manuscript and approved it for publication.

\section{Compliance with ethical standards}

Conflict of interest The authors declare that they have no conflict of interest.

Ethical approval This study was approved by Ethics Commission of First Hospital of Yangtze University, Clinical medical college, Yangtze University, Jingzhou, Hubei, 434000, China.

Informed consent Written informed consent was waived by the Ethics Commission of the designated hospital for emerging infectious diseases.

\section{References}

1. Zhu N, Zhang D, Wang W, Li X, Yang B, Song J, Zhao X, Huang B, Shi W, Lu R, Niu P, Zhan F, Ma X, Wang D, Xu W, Wu G, Gao GF, Tan W (2020) A novel coronavirus from patients with pneumonia in China, 2019. N Engl J Med 382(8):727-733. https://doi.org/10.1056/NEJMoa2001017

2. Patel A, Jernigan DB, 2019-nCoV CDC Response Team (2020) Initial public health response and interim clinical guidance for the 2019 Novel Coronavirus outbreak-United States, December 31, 2019-February 4, 2020. MMWR. Morbidity and mortality weekly report 69(5):140-146

3. Chen L, Li X, Chen M, Feng Y, Xiong C (2020) The ACE2 expression in human heart indicates new potential mechanism of heart injury among patients infected with SARS-CoV-2. Cardiovasc Res 116(6):1097-1100. https://doi.org/10.1093/cvr/cvaa0 78

4. Santos RA, Ferreira AJ, Simões ESAC (2008) Recent advances in the angiotensin-converting enzyme 2-angiotensin(1-7)-Mas axis. Exp Physiol 93(5):519-527. https://doi.org/10.1113/expph ysiol.2008.042002

5. Hamming I, Timens W, Bulthuis ML, Lely AT, Navis G, van Goor H (2004) Tissue distribution of ACE2 protein, the functional receptor for SARS coronavirus. A first step in understanding SARS pathogenesis. J Pathol 203(2):631-637. https://doi. org/10.1002/path.1570

6. Danilczyk U, Penninger JM (2006) Angiotensin-converting enzyme II in the heart and the kidney. Circ Res 98(4):463-471. https://doi.org/10.1161/01.RES.0000205761.22353.5f

7. Bernardi S, Toffoli B, Zennaro C, Tikellis C, Monticone S, Losurdo P, Bellini G, Thomas MC, Fallo F, Veglio F, Johnston CI, Fabris B (2012) High-salt diet increases glomerular ACE/ACE2 ratio leading to oxidative stress and kidney damage. Nephrol Dial Transplant 27(5):1793-1800. https://doi. org/10.1093/ndt/gfr600

8. Wu F, Zhao S, Yu B, Chen YM, Wang W, Song ZG, Hu Y, Tao ZW, Tian JH, Pei YY, Yuan ML, Zhang YL, Dai FH, Liu Y, Wang QM, Zheng JJ, Xu L, Holmes EC, Zhang YZ (2020) A new coronavirus associated with human respiratory disease in China. Nature 579(7798):265-269. https://doi.org/10.1038/ s41586-020-2008-3
9. Reynolds RM, Padfield PL, Seckl JR (2006) Disorders of sodium balance. BMJ (Clin Res Ed) 332(7543):702-705. https ://doi.org/10.1136/bmj.332.7543.702

10. Adrogué HJ, Madias NE (2000) Hyponatremia. N Engl J Med 342(21):1581-1589. https://doi.org/10.1056/nejm20000525342 2107

11. Verbrugge FH, Steels P, Grieten L, Nijst P, Tang WH, Mullens W (2015) Hyponatremia in acute decompensated heart failure: depletion versus dilution. J Am Coll Cardiol 65(5):480-492. https://doi.org/10.1016/j.jacc.2014.12.010

12. Ali Q, Patel S, Hussain T (2015) Angiotensin AT2 receptor agonist prevents salt-sensitive hypertension in obese Zucker rats. Am J Physiol Renal Physiol 308(12):F1379-1385. https://doi. org/10.1152/ajprenal.00002.2015

13. Aggarwal S, Garcia-Telles N, Aggarwal G, Lavie C, Lippi G, Henry BM (2020) Clinical features, laboratory characteristics, and outcomes of patients hospitalized with coronavirus disease 2019 (COVID-19): Early report from the United States. Diagnosis 7(2):91-96. https://doi.org/10.1515/dx-2020-0046

14. Berni A, Malandrino D, Parenti G, Maggi M, Poggesi L, Peri A (2020) Hyponatremia, IL-6, and SARS-CoV-2 (COVID-19) infection: may all fit together? J Endocrinol Invest 43(8):11371139. https://doi.org/10.1007/s40618-020-01301-w

15. Lippi G, South AM, Henry BM (2020) Electrolyte imbalances in patients with severe coronavirus disease 2019 (COVID-19). Ann Clin Biochem. https://doi.org/10.1177/000456322092225 5

16. Post A, Dullaart RPF, Bakker SJL (2020) Is low sodium intake a risk factor for severe and fatal COVID-19 infection? Eur J Intern Med 75:109. https://doi.org/10.1016/j.ejim.2020.04.003

17. National Health Commission of the people's republic of China (2020) New-coronavirus-pneumonia-prevention-and-controlprogram (in Chinese) 7th ed https://www.nhc.gov.cn/yzygj/ s7653p/202003/46c9294a7dfe4cef80dc7f5912eb1989/files/ ce3e6945832a438eaae415350a8ce964.pdf.

18. Sterns RH (2015) Disorders of plasma sodium-causes, consequences, and correction. N Engl J Med 372(1):55-65. https:// doi.org/10.1056/NEJMra1404489

19. Klein L, O'Connor CM, Leimberger JD, Gattis-Stough W, Piña IL, Felker GM, Adams KF Jr, Califf RM, Gheorghiade M (2005) Lower serum sodium is associated with increased short-term mortality in hospitalized patients with worsening heart failure: results from the Outcomes of a Prospective Trial of Intravenous Milrinone for Exacerbations of Chronic Heart Failure (OPTIME-CHF) study. Circulation 111(19):2454-2460. https ://doi.org/10.1161/01.cir.0000165065.82609.3d

20. Yousaf Z, Al-Shokri SD, Al-Soub H, Mohamed MFH (2020) COVID-19-associated SIADH: a clue in the times of pandemic! Am J Physiol Endocrinol Metab 318(6):E882-e885. https://doi. org/10.1152/ajpendo.00178.2020

21. Gemcioglu E, Karabuga B, Ercan A, Erden A (2020) A case of inappropriate antidiuretic hormone secretion syndrome associated with covid-19 pneumonia. Acta Endo (Buc). 16(1):110 111. https://doi.org/10.4183/aeb.2020.110

22. Ravioli S, Niebuhr N, Ruchti C, Pluess E, Stoeckli T, Lindner G (2020) The syndrome of inappropriate antidiuresis in COVID19 pneumonia: report of two cases. Clin Kidney J 13(3):461462. https://doi.org/10.1093/ckj/sfaa080

23. Khalangot M (2020) COVID-19 and SIADH relations: impact of the positive pressure ventilation. Am J Physiol Endocrinol Metab 319(1):E196. https://doi.org/10.1152/ajpendo.00288 .2020

24. Jones DP (2018) Syndrome of inappropriate secretion of antidiuretic hormone and hyponatremia. Pediatr Rev 39(1):27-35. https://doi.org/10.1542/pir.2016-0165 
25. Li H, Huang L, Wu G, Chen X, Zheng Q, Su F, Liang M, Chen $X$ (2019) Be aware of the effects of glucocorticoids on SIADH: A case report. Medicine 98(7):e14295. https://doi.org/10.1097/ md.0000000000014295

26. Kumar V, Malhotra I, Shankar S, Kupfer Y (2018) Syndrome of inappropriate antidiuretic hormone consecutive to treatment with ciprofloxacin. Am J Ther 25(6):e684-e685. https://doi. org/10.1097/mjt.0000000000000642
Publisher's Note Springer Nature remains neutral with regard to jurisdictional claims in published maps and institutional affiliations. 